\title{
Long-term results of pulmonary artery rehabilitation in patients with pulmonary atresia, ventricular septal defect, pulmonary artery hypoplasia, and major aortopulmonary collaterals
}

\author{
Andreea Dragulescu, MD, PhD, ${ }^{a}$ Issam Kammache, MD, ${ }^{a}$ Virginie Fouilloux, MD, ${ }^{\mathrm{b}}$ Pascal Amedro, MD, \\ Dominique Métras, $\mathrm{MD},{ }^{\mathrm{a}}$ Bernard Kreitmann, $\mathrm{MD}, \mathrm{PhD},{ }^{\mathrm{b}}$ and Alain Fraisse, $\mathrm{MD}, \mathrm{PhD}^{\mathrm{a}}$
}

\begin{abstract}
Objective: The study objective was to report the long-term results of pulmonary artery rehabilitation in patients with pulmonary atresia, ventricular septal defect, hypoplastic pulmonary arteries, and major aortopulmonary collaterals.
\end{abstract}

\begin{abstract}
Methods: Since 1991, 20 patients with profound pulmonary artery hypoplasia (mean Nakata index $26 \pm 14$ $\mathrm{mm}^{2} / \mathrm{m}^{2}$ ) have undergone a medico-surgical strategy of native pulmonary artery rehabilitation to achieve complete repair with satisfactory hemodynamics (right ventricle to aortic pressure ratio $<0.8$ ).

Results: The first step, right ventricle to pulmonary artery connection, was performed at a median age of 4.1 (0.1-18.7) months with 1 operative death. After a median duration of 4.3 (1.1-26) months, the second step of interventional catheterizations followed (median, 2 (1-7)/patient), consisting of 36 pulmonary angioplasties, 11 stent implantations, and 20 collateral occlusions. Significant pulmonary artery growth was obtained in all cases with a Nakata index of $208 \pm 85 \mathrm{~mm}^{2} / \mathrm{m}^{2}$ before surgical correction $(P<.001)$. The third step of surgical repair was performed at a median age of $1.9(0.6-10.7)$ years, with right ventricular outflow reconstruction and ventricular septal defect closure fenestrated in 3 cases. During a mean follow-up of $8.2 \pm 4.5$ years, pulmonary artery rehabilitation was pursued in most patients, with 47 pulmonary angioplasties, 15 stent implantations, and 11 collateral occlusions. Three patients with a poor hemodynamic result died. At last visit, the 16 survivors are in New York Heart Association class I $(n=12)$ or II $(n=4)$ with satisfactory hemodynamics in 13 cases.
\end{abstract}

Conclusions: Pulmonary artery rehabilitation allows complete repair in the majority of patients with pulmonary atresia, ventricular septal defect, hypoplastic pulmonary arteries, and major aortopulmonary collaterals. However, long-term management often requires pursuit of the rehabilitation process. (J Thorac Cardiovasc Surg $2011 ; 142: 1374-80)$

Pulmonary atresia with ventricular septal defect (VSD), diminutive but confluent pulmonary arteries (PAs), and major aortopulmonary collateral arteries (MAPCAs) is a rare and complex lesion with great morphologic variability regarding the pulmonary blood flow. This is entirely dependent on MAPCA number, size, course, origin, distribution, and connections with native PAs. ${ }^{1}$ MAPCAs most often arise from the descending aorta, and in most cases, duplicate pulmonary blood flow can be identified from collaterals and central PAs even if collateral wash-in/ wash-out flow is difficult to interpret from a distribution point of view. ${ }^{2}$

From the Cardiologie Pédiatrique, ${ }^{\mathrm{a}}$ Hôpital de la Timone-Enfants, Marseille, France; Service de Chirurgie Thoracique et Cardiovasculaire, ${ }^{\mathrm{b}}$ Hôpital d'Enfants de la Timone, Pierre, Marseille, France; and Service de Pédiatrie 1, ${ }^{\mathrm{c}}$ Hôpital Arnaud de Villeneuve, Montpellier, France.

Disclosures: Authors have nothing to disclose with regard to commercial support.

Received for publication Sept 29, 2010; revisions received March 9, 2011; accepted for publication May 18, 2011; available ahead of print July 1, 2011.

Address for reprints: Alain Fraisse, MD, PhD, Cardiologie Pédiatrique, Hôpital de la

Timone-Enfants, 264 rue St Pierre, 13385 Marseille Cedex 5, France (E-mail:

alain.fraisse@ap-hm.fr).

0022-5223/\$36.00

Copyright (c) 2011 by The American Association for Thoracic Surgery

doi:10.1016/j.jtcvs.2011.05.010
There are 2 major strategies to approach these patients. One is characterized by using MAPCAs for unifocalization in 1 or 2 steps toward surgical repair. ${ }^{1,3-5}$ However, the histopathologic structure of MAPCAs predisposes them to multilevel stenosis and thrombosis. Consequently, patients often experience poor hemodynamic result after repair. ${ }^{5,6}$ The other strategy is focused on the rehabilitation of native PAs with an initial ascending aorta to PA connection ${ }^{7}$ or right ventricle (RV) to PA connection to promote native PA growth. ${ }^{8}$ Once the antegrade flow is established, there is an easier approach for interventional PA dilation to relieve stenosis and to further promote growth. It also allows subsequent occlusion of collaterals that are redundant. When PA development is judged adequate, surgical repair is performed.

However, reports on such a strategy of native PA rehabilitation are scarce, and its long-term results with clinical and hemodynamic evaluation remains unknown. ${ }^{8-10}$ Moreover, previous reports that demonstrated the feasibility of such strategy did not provide the precise timing and description of different steps.

To achieve these goals, we retrospectively reviewed our institutional experience with pulmonary atresia, VSD, hypoplastic "seagull" PAs, and MAPCAs. 


$$
\begin{aligned}
& \text { Abbreviations and Acronyms } \\
& \qquad \begin{aligned}
\text { MAPCA }= & \text { major aortopulmonary collateral } \\
& \text { artery } \\
\text { PA } & \text { pulmonary artery } \\
\text { RV } & =\text { right ventricle } \\
\text { VSD } \quad= & \text { ventricular septal defect }
\end{aligned}
\end{aligned}
$$

\section{MATERIALS AND METHODS}

This is a retrospective, monocentric study. Since 1991, we identified 20 patients with pulmonary atresia, VSD, hypoplastic "seagull" PAs (Nakata index $<90 \mathrm{~mm}^{2} / \mathrm{m}^{2}$ ), and MAPCAs (Figure 1). We excluded patients with duct-dependent pulmonary circulation and patients with discontinuous PAs. Patients with hypoplastic PAs, MAPCAs, and a ductus arteriosuslike collateral were included if no prostaglandin E1 infusion was needed. Descriptions of 10 and 17 of the 20 patients were published in 2 preliminary descriptive studies. ${ }^{9,10}$ The study protocol was reviewed and approved by the local institutional ethic committee.

\section{Multistage Pulmonary Artery Rehabilitation Strategy}

All patients with this anatomy referred to our institution during the study period underwent this PA rehabilitation strategy that was first described at Children's Hospital, Boston. ${ }^{8}$ We further developed the 3 steps with a clear timing definition:

1. RV-PA connection is performed as early as possible, usually in the first 6 months of life, to favor antegrade flow and potentially to promote angiogenesis. $^{8}$

2. Starting 3 to 5 months after RV-PA connection, catheter interventions are performed to address PA stenosis or hypoplasia and to occlude MAPCAs in all areas of dual pulmonary blood supply, with the goal to favor antegrade perfusion and development of distal PAs. MAPCAs as the only source of blood supply for pulmonary segments or lobes are maintained.

3. Patients are considered suitable for surgical repair according to the following criteria: clinical signs of left-to-right shunting or a transcutaneous oxygen saturation of $80 \%$ or more; native PAs have to reach an acceptable growth (Nakata index $\geq 90 \mathrm{~mm}^{2} / \mathrm{m}^{2}$ ); and all significant redundant MAPCAs are closed. In rare cases with a proximal connection between MAPCAs and native PAs, transcatheter occlusion may compromise native PAs. Such MAPCAs are surgically closed at the time of repair.

A complete repair with VSD closure, PA reconstruction, and valved RVPA conduit is accomplished.

The hemodynamic result is considered acceptable if RV to aortic pressure ratio is less than 0.8 , with distal mean PA pressure less than 25 mm Hg. ${ }^{8}$

\section{Patient Characteristics}

Demographic and morphologic data are presented in Table 1.

\section{Native Nakata index}

The Nakata index was calculated using the following formula: right PA cross-sectional area in millimeters squared + left PA cross-sectional area in millimeters squared/body surface area in meters squared. The PAs were measured before branching, and the result was expressed in millimeters squared/meters squared (branch PA area indexed to body surface area). ${ }^{11}$ The collateral arteries were not included in the equation.

\section{Cardiac Catheterization}

Initial diagnostic evaluation. A complete description of the pulmonary vasculature was performed in all patients, paying particular attention to identify all MAPCAs and to obtain an angiogram of the "seagull." If the native PA was not visualized through the collaterals, a wedge pulmonary vein angiogram was performed.

\section{Interventional Procedures (Step 2)}

Interventional catheterizations were performed in each patient under general anesthesia. The major issues addressed were stenosis/hypoplasia of native PAs, obstruction of RV-PA connection, or residual MAPCAs. The hemodynamic evaluation included measurements of distal PA pressure in different segments, RV to aorta pressure ratio, and pressure gradients in the pulmonary tree. The criterion for balloon dilation was the presence of significant angiographic stenosis. The only exception was residual proximal native PA stenosis when the patient's Nakata index was satisfactory enough to allow surgical repair, because such stenosis would be surgically repaired during the third step. Balloon angioplasty was always attempted with high-pressure balloons, followed by stent implantation in case of estimated poor result. Since 2003, cutting balloons were used in stenotic vessels resistant to high-pressure balloon angioplasty. ${ }^{12}$ Success for balloon dilation and stenting based on Rothman and colleagues ${ }^{12}$ criteria was an increase in vessel diameter greater than $50 \%$; the 2 other criteria from Rothman and colleagues ( $>20 \%$ increase in flow to the dilated lung, $20 \%$ decrease in $\mathrm{RV} /$ systemic pressure ratio) were not applicable in the setting of multiple peripheral PA stenosis, unrestrictive intracardiac, and extracardiac shunts. ${ }^{13}$ MAPCA occlusion was performed, paying particular attention to position coils as proximal as possible to avoid native PA occlusion (Figure 1). Our aim was to relieve as much of the stenosis as possible, especially for the distal branches using high-pressure balloons and cutting balloons for the intrahilar arteries. Procedures were repeated until PA growth was considered satisfactory to allow surgical repair.

\section{Surgical Approaches}

Step 1: Right ventricle-pulmonary artery connection. Patients were operated via a median sternotomy with the use of normothermic cardiopulmonary bypass on a beating heart, as already described. ${ }^{9,10}$ After controlling the right and left PAs with gentle occlusion by rubber loops, the main PA was incised longitudinally, and an augmentation small oval patch of autologous pericardium prepared with glutaraldehyde was sutured to the edges of arteriotomy. The incision was than extended to the RV epicardium, and the patch was sewn to the edges of this incision. Finally, the RV infundibulum was opened, and the patch insertion was completed (Figure 2), making sure the opening in the RV was sufficient by resection of hypertrophied muscle and spreading this opening with scissors. During this part of the procedure, prevention of air embolism was achieved using a small suction catheter in the ascending aorta, just proximal to a partial crossclamping (bubble trap) and by avoiding entering the pump sucker in the RV cavity. Alternatively, an initial central shunt between the ascending aorta and the PA was performed in 3 patients.

Step 3: Surgical repair. This consisted of a redo-sternotomy and complete repair under cardiopulmonary bypass. A reconstruction of the pulmonary bifurcation and branches (as far as surgically possible) was performed after aortic transection. The PA bifurcation and branches (usually intrahilar) were reconstructed using homograft patches in the majority of patients. When necessary for branch PA reconstruction, the proximal deployed stents were cut or removed. In some cases, the distal PA plasty was performed during short periods of hypothermic circulatory arrest for better visualization. For the RV-PA continuity, a cryopreserved homograft conduit was used in most patients and a monovalved patch was used in some patients. The patch VSD closure using Dacron material was 


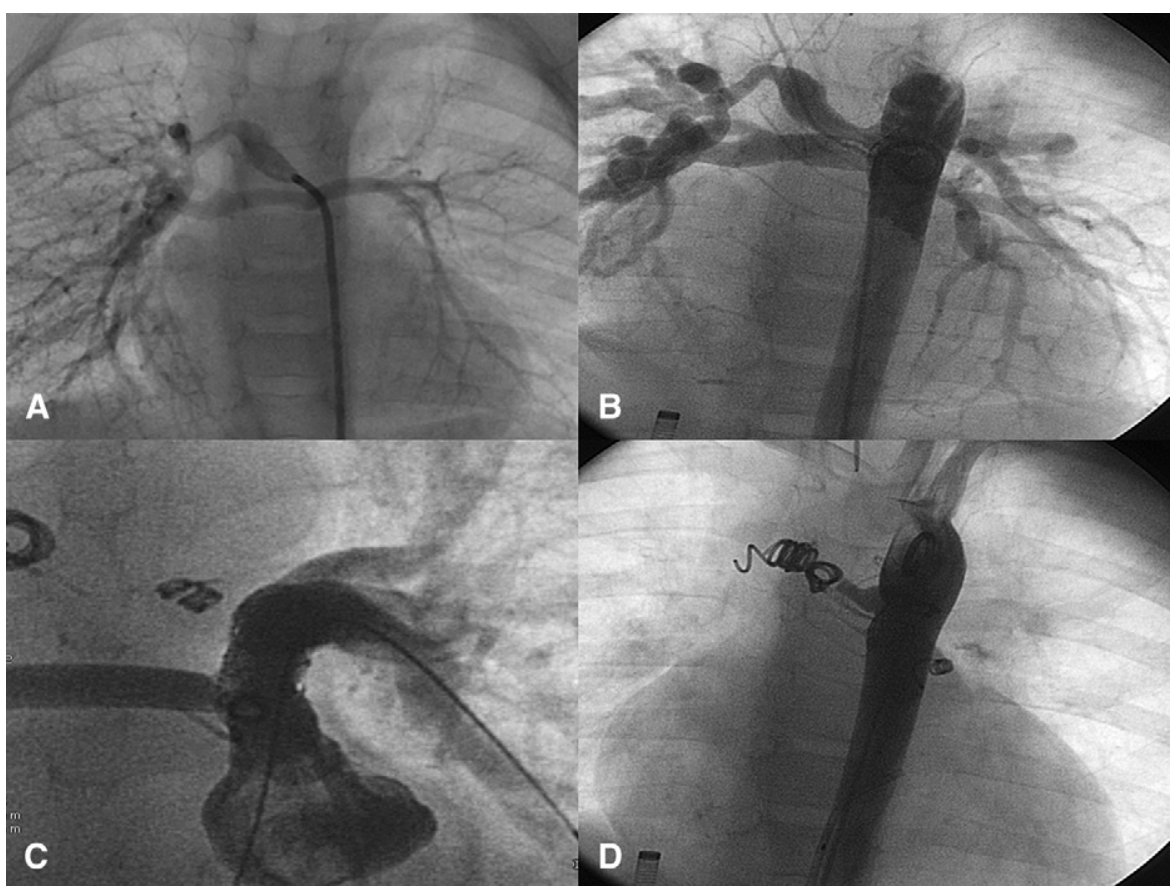

FIGURE 1. Initial angiogram through a right-sided MAPCA in an 11-month-old infant showing hypoplasia (Nakata index at $\left.34 \mathrm{~mm} / \mathrm{m}^{2}\right)$ of native PA (A), with pulmonary arterial blood flow mainly dependent on the MAPCAs in the aortogram (B). Twelve months after right-ventricle to PA surgical connection and 3 months after the last transcatheter rehabilitation with left PA stenting, the native PA bed achieved appropriate growth with a Nakata index of $187 \mathrm{~mm}^{2} /$ $\mathrm{m}^{2}(\mathrm{C})$, and all the MAPCAs have been occluded proximally (D), allowing subsequent surgical repair.

completed by transatrial or transventricular approach, with elective fenestration if necessary. At the atrial level, a small foramen ovale was left open or created. At the completion of cardiopulmonary bypass, pressures were recorded, and if the RV/left ventricle pressure ratio was less than 1, the correction was considered satisfactory, and the VSD was left closed.

\section{Statistical Analysis}

Group statistics are expressed as mean \pm standard deviation or median (minimum-maximum) when appropriate. Wilcoxon paired test was used to compare the Nakata index of the native PAs before and after second-step rehabilitation. To identify the risk factors for right ventricular failure and death, we used Fischer exact tests for categoric variables and Mann-Whitney tests for ordinal or continuous variables. The following variables as risk factors for post-repair death and poor hemodynamic result were analyzed: sex, 22q11 microdeletion, initial Nakata index, number of MAPCA, age at RV-PA connection, type of RV-PA connection, oxygen saturation after RV-

TABLE 1. Demographic data and morphologic features at presentation

\begin{tabular}{lc}
\hline \multicolumn{1}{c}{ Characteristics } \\
\hline Male/female & $12 / 8$ \\
Microdeletion 22q11 & 7 \\
Mean Nakata index \pm SD & $26 \pm 14 \mathrm{~mm}^{2} / \mathrm{m}^{2}$ \\
Mean $\mathrm{O}_{2}$ saturation $\pm \mathrm{SD}$ & $76 \% \pm 5 \%$ \\
No. of MAPCAs per patient & $4(2-6)$ \\
Right aortic arch & 7 \\
Lusoria (right subclavian artery) & 1 \\
Left superior vena cava & 3 \\
Additional VSD & 1 \\
\hline
\end{tabular}

$\mathrm{O}_{2}$ saturation, transcutaneous oxygen saturation; $S D$, standard deviation.
PA connection, total interventional catheterizations, fenestrated VSD patch, and unifocalization. The statistical analysis was performed using SPSS version 15.0 for Windows (SPSS Inc, Chicago, Ill).

\section{RESULTS \\ Step 1}

RV-PA connection was performed at a mean age and weight of $5.7 \pm 4.3$ months and $5.6 \pm 2.1 \mathrm{~kg}$, respectively.

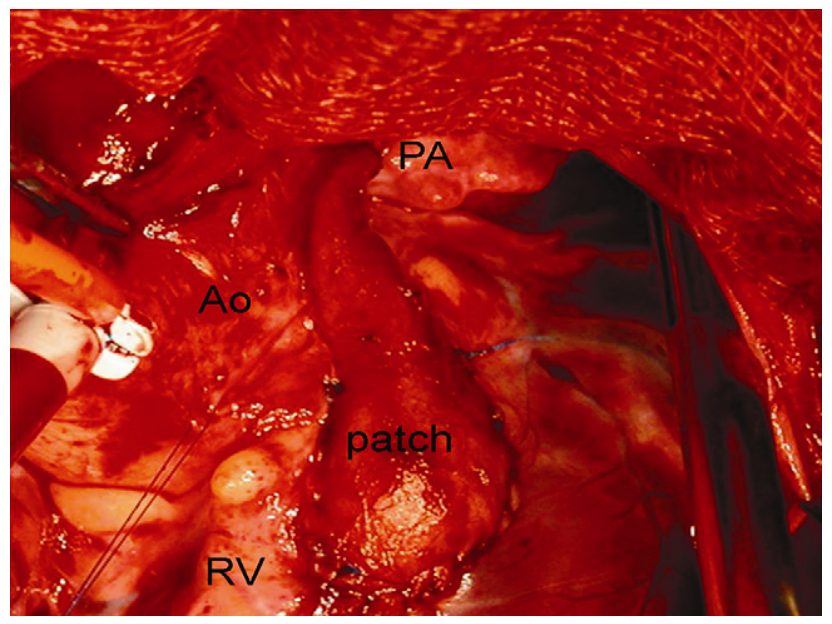

FIGURE 2. RV to PA connection using pericardial patch, through median sternotomy as the first step of the rehabilitation strategy. Ao, Aorta; $P A$, pulmonary artery; $R V$, right ventricle. 
This was followed by a mean improvement in transcutaneous oxygen saturation of $11 \% \pm 5 \%$. One patient who underwent emergency surgery at 2 days of life died immediately after RV-PA connection with severe cyanosis. Three patients had an initial central shunt between the ascending aorta and the PA; in 2 cases, this was performed in another hospital, the patients being addressed afterward. All of them presented with severe cyanosis and underwent a second surgery of RV-PA connection because the PAs remained diminutive with an autologous-treated pericardial patch in 2 patients and a small homograft in 1 patient. Because of rapid stenosis of the homograft in the latter case, reoperation was performed 2 months later with autologous pericardial patch augmentation.

\section{Step 2}

After a mean interval of $5.8 \pm 4.5$ months after RV-PA connection, all patients underwent a first interventional cardiac catheterization. A median of 2 (1-7) interventional catheterizations per patient was performed during this second step. Interventional procedures consisted of 36 balloon angioplasties with 11 stent placements and 20 MAPCA occlusions (Figure 1). In 2 patients, balloon dilation of the RVPA connection was performed because of severe stenosis. One patient had a stent placed on the proximal left PA that extended retrogradely to the RV-PA connection. Although transcatheter access to the right PA was potentially compromised because of the stent position, there was no further indication to balloon dilate or stent the right PA.

The Nakata index of the native PAs increased significantly to $209 \pm 85 \mathrm{~mm}^{2} / \mathrm{m}^{2}(P<.001)$ as shown in Figure 3. There were no deaths.

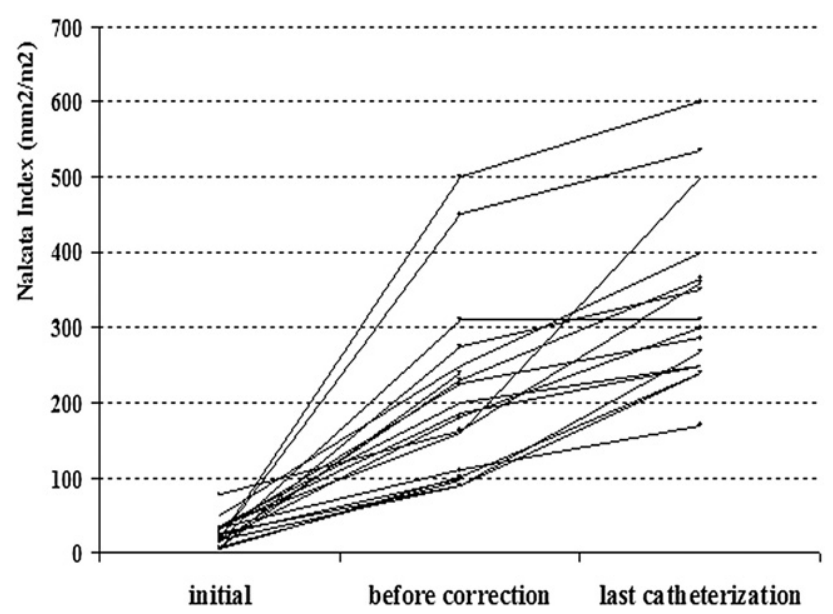

FIGURE 3. Nakata index increase of native PAs during rehabilitation process. The initial Nakata index was $26 \pm 14 \mathrm{~mm}^{2} / \mathrm{m}^{2}$, increased at $208 \pm 85$ $\mathrm{mm}^{2} / \mathrm{m}^{2}$ after the second step of transcatheter rehabilitation and before surgical repair, with a further increase up to $330 \pm 89 \mathrm{~mm}^{2} / \mathrm{m}^{2}$ at the last catheterization.

\section{Step 3}

At a mean age of $2.4 \pm 2.3$ years, surgical repair was performed in 19 patients. Their clinical, hemodynamic, and angiographic data are presented in Table 2. Fifteen patients had clinical evidence of left-to-right shunting and satisfactory criteria for PA growth, as defined in "Materials and Methods." In 4 patients with a satisfactory Nakata index but profound cyanosis (numbers 2, 4, 12, and 16; Table 2), surgical repair was performed because pulmonary blood flow was mainly limited by proximal native PA stenosis that could be surgically addressed. A valved conduit was used for the reconstruction of the RV outflow tract in 15 cases (11 pulmonary homografts, 3 aortic homografts, and 1 Contegra tube). Four patients had a monovalved patch. Unilateral unifocalization of MAPCAs supplying pulmonary segments without native PA branching was performed in 4 patients. Four MAPCAs with a proximal connection with native PAs could not be closed percutaneously during the second step and were surgically ligated. In 3 patients, the VSD patch was electively fenestrated with a 4-mm hole, and in 1 patient an additional small muscular VSD was left open. The 3 patients who preoperatively had distal pulmonary arterial hypertension (mean PAP $\geq 25 \mathrm{~mm} \mathrm{Hg}$ ) in the setting of left-to-right shunting did not experience postoperative pulmonary hypertension crisis. No patient died during the postoperative period.

TABLE 2. Clinical and hemodynamic data at surgical repair (step 3) $\mathrm{O}_{2}$

\begin{tabular}{lcccccc}
$\begin{array}{c}\text { Patient } \\
\text { no. }\end{array}$ & $\begin{array}{c}\text { Weight } \\
(\mathbf{k g})\end{array}$ & $\begin{array}{c}\text { Age } \\
(\mathbf{y})\end{array}$ & $\begin{array}{c}\text { saturation* } \\
(\%)\end{array}$ & CHF & mPAP & $\begin{array}{c}\text { Nakata } \\
\left(\mathbf{m m}^{\mathbf{2}} / \mathbf{m}^{\mathbf{2}}\right)\end{array}$ \\
\hline 1 & 10 & 2.6 & 85 & & 20 & 240 \\
2 & 11.7 & 3.7 & 78 & & 15 & 97 \\
3 & 8 & 1.5 & 85 & + & 32 & 450 \\
4 & 13.2 & 3.2 & 77 & & 14 & 110 \\
5 & 11 & 2.5 & 83 & & 19 & 230 \\
6 & 8 & 0.9 & 80 & & 10 & 180 \\
7 & 15 & 4.1 & 86 & & 12 & 225 \\
8 & 6.8 & 0.9 & 83 & & 22 & 275 \\
9 & 6 & 0.5 & 90 & & 21 & 100 \\
10 & 14 & 3.4 & 98 & & 10 & 110 \\
11 & 6.4 & 0.7 & 95 & + & 15 & 95 \\
12 & 10.3 & 2.3 & 75 & & 17 & 90 \\
13 & 28 & 10.7 & 94 & + & 34 & 310 \\
14 & 9.3 & 2.1 & 85 & + & 17 & 500 \\
15 & 9.6 & 1.3 & 91 & & 22 & 163 \\
16 & 9 & 1.6 & 74 & & 11 & 160 \\
17 & 7.8 & 0.6 & 84 & & 35 & 200 \\
18 & 7.5 & 0.9 & 86 & & 16 & 250 \\
19 & 10 & 2.3 & 85 & + & 9 & 187 \\
\hline$O_{2}$ & & & & &
\end{tabular}

$\mathrm{O}_{2}$ saturation, Transcutaneous oxygen saturation; $\mathrm{CHF}$, congestive heart failure; mPAP, mean distal pulmonary artery pressure. *Transcutaneous oxygen saturation. 


\section{Follow-up}

The mean follow-up after surgical correction was $8.2 \pm$ 4.5 years. Three patients died $0.3,0.4$, and 11.8 years after surgical repair in a clinical setting of RV failure. The first patient presented with severe respiratory distress and fever 4 months after discharge. The abdomen was distended with large hepatomegaly. He experienced cardiac arrest in the emergency department and could not be resuscitated. The second patient died during an interventional catheterization with PA stenting 5 months after correction, in the setting of acute RV failure and arrhythmia with extensive thrombosis of the caval system. The third patient had a rupture of follow-up for 10 years and died 11.8 years after repair as a result of severe RV dysfunction despite surgical reintervention for RV-PA conduit replacement. RV wall biopsy during surgery showed almost complete fibrosis and scarce cardiomyocytes.

Because of significant branch PA stenosis or elevated RV pressure in the setting of multiple peripheral pulmonary stenosis, most of the patients benefited from further interventions (catheterizations or surgery) during follow-up, as a continuum of the rehabilitation process. Until the last follow-up, a median of $3(0-7)$ transcatheter interventions per patient were performed, consisting of 52 balloon angioplasties with 17 stent placements and 12 residual collateral occlusions. Three patients underwent reoperation with RVPA conduit replacement and PA enlargement at 2.2, 5.6, and 11.8 years after correction. Subsequent significant PA growth $(P<.001)$ was obtained with a mean Nakata index of $330 \pm 89 \mathrm{~mm}^{2} / \mathrm{m}^{2}$ at last transcatheter evaluation (Figure 3), when the median RV to aortic pressure ratio was $0.58(0.35-1)$. Two of the survivors had a poor hemodynamic result (ratio $>0.8$ ) with obstructive pulmonary vascular disease.

In univariate analysis there was no significant risk factor for postsurgical repair death or poor hemodynamic result. At last follow-up, all patients were in good clinical condition, 14 patients were in New York Heart Association class 0/I, and 2 patients were in New York Heart Association class II. Median oxygen saturation was $96 \%(88 \%-$ $100 \%$ ) with 3 patients having a trivial, nonsignificant left to right residual shunt at the ventricular level.

\section{DISCUSSION}

The management of patients with pulmonary atresia, VSD, diminutive PA, and MAPCA represents a challenge with several reported strategies. ${ }^{1,3-10,14,15}$ Unifocalization of MAPCAs and native PAs grants surgical correction in the majority of cases, with good postoperative survival, but no long-term results have been published. ${ }^{3,14,15}$ There are reports suggesting that unifocalized MAPCAs show a high incidence of occlusion and no growth, ${ }^{5,6}$ which is concordant with our experience of poor evolution of unifocalized collaterals. Central end-to-side aortopulmonary shunt also promotes native PA growth, but in a recent report by Mumtaz and colleagues ${ }^{7}$ only $60 \%$ of the patients achieved a complete repair. One of the inconveniences of this strategy is that transcatheter PA dilatation is more difficult in these patients, requiring a retrograde approach through the aorta.

The concept of PA rehabilitation has emerged in the mid1980 s with the pioneering work of the Boston group. ${ }^{8}$ One of the main premises was to consider that providing antegrade flow to the native PAs promotes growth of these arteries but also allows a better evaluation of their effective distribution to different pulmonary segments, because dual perfusion is present in most of the cases. This is enhanced by a more recent pathologic study that demonstrates that the majority of shunted patients with deeply cyanotic congenital heart disease present severe intrinsic pulmonary arteriopathy with decreased PA growth potential. ${ }^{16} \mathrm{Also}$, on the basis of our experience, this group of patients with hypoplastic but confluent PAs have a complete or almost complete distribution to lung segments with seldom the need for segmental unifocalization at the time of surgical repair.

Even if results of the Boston group were promising with successful repair in a large percentage of patients and better outcome than the other strategies, the overall mortality rate was still high, and there were several issues that remained unsolved. By reporting the initial experience with a relatively small number of patients, there was no clear stage definition and no optimum timing for each stage. ${ }^{8}$ In the present study, we describe a precise staged approach with early RV-PA connection, ideally within the first 6 months of life, followed by catheter interventions to address PA stenosis or hypoplasia and collateral occlusion. Because balloon angioplasty at a recent surgical suture line is expected to increase the risk for PA rupture, ${ }^{17}$ we usually wait 2 months after RV-PA connection. Moreover, this allows the patient to grow and achieve a point where catheter interventions can be performed with lower risk. After this time period, native PA stenosis angioplasties and concomitant MAPCAs occlusions are rapidly performed because we speculate that the development of native PA stenosis is due to the opposing flows through the RVOT and MAPCAs, producing watershed areas in the native PAs. The surgical correction is performed once the PA development is considered satisfactory, usually within 1 year after RV-PA connection. The rehabilitation of native PAs has come to a complete anatomic correction and long-time survival in $80 \%$ of patients. We emphasize the importance of the process of rehabilitation as a continuum, because further interventions were necessary during follow-up in the majority of our patients to maintain satisfactory hemodynamics and PA growths.

The process of rehabilitation allows the development of native PA with a 10 -fold increase of Nakata index before 
surgical correction. As previously reported ${ }^{8,9}$ PA growth was considered satisfactory if the Nakata index exceeded $150 \mathrm{~mm}^{2} / \mathrm{m}^{2}$, which was the case in the majority of our study patients. Patients with a Nakata index between 90 and $150 \mathrm{~mm}^{2} / \mathrm{m}^{2}$ were still suitable for surgical repair but may represent a higher risk group, although successful repair was also obtained in 3 patients with a Nakata index less than $100 \mathrm{~mm}^{2} / \mathrm{m}^{2}$, with wide patching of the pulmonary bifurcation and proximal branches in 2 and an electively fenestrated VSD patch in 1. Two other patients with residual distal PA stenosis also had an elective fenestrated VSD to protect the postoperative RV from suprasystemic pressures. Such strategy is supported by a recent study of Marshall and colleagues ${ }^{18}$ showing that elective use of fenestrated patch for VSD closure in selected patients with diminutive PA and VSD improves survival with a low incidence of significant left to right shunt. Conversely, another study reports that the need to reopen the VSD in the operating room because of unfavorable hemodynamics is predictive of both early and late mortality. ${ }^{19}$ All this suggests that in highrisk patients with profoundly diminutive native PAs or multiple and severe distal stenosis, it is better to perform elective VSD fenestration than to consider it on an emergency basis during or after bypass weaning in the setting of unfavorable hemodynamics. All our 3 patients with fenestrated VSD survived, with a poor hemodynamic result in 2 of them with severe residual peripheral PA stenosis and more favorable hemodynamics with a nonsignificant left to right shunt in one.

We and others have observed that patients with pulmonary atresia-VSD experience markedly abnormal development in the proximal and distal pulmonary vascular bed with multiple distortion and stenosis. ${ }^{8,9,16}$ Thus, interventional catheterization with balloon dilatation \pm stenting of PAs is virtually indicated in all the cases. This allows a good hemodynamic result to be preserved in the majority of them. Moreover, successful balloon angioplasty of residual PA stenosis has been shown to improve exercise capacity (peak oxygen consumption and gas exchange) by improving pulmonary blood flow distribution and reducing ventilation/perfusion mismatch. ${ }^{20}$ All this supports the necessity to relieve as early as possible residual obstruction. The use of stents even in small children is efficient. Redilation of previously implanted stents is feasible and successful in case of previous elective underexpansion, obstructive neointimal proliferation, and growth that resulted in relative stenosis. ${ }^{21}$ Finally, development of pulmonary vascular disease in this group of patients is variable and probably diminishes with aggressive management, allowing surgical repair early in life. Still, peripheral stenosis not addressed invasively may favor other segments' exposure to high pressures and further development of vascular disease.

\section{Study Limitations}

This study has the inherent limitations of its retrospective nature. The rehabilitation strategy we describe is only valid for patients with hypoplastic but confluent native PAs. Patients with discontinuous PAs were excluded from the present study because their management is not based on RV-PA connection followed by transcatheter rehabilitation. Although a study ${ }^{22}$ reported that MAPCAs can coexist with hypoplastic and discontinuous PAs in $30 \%$ of the cases, such cases are less frequently encountered in our experience. We speculate this is because the patients referred to us are managed early in life. By diagnostic cardiac catheterization with exhaustive pulmonary vascular bed angiograms (including pulmonary vein wedge angiograms), we almost always documented confluent central PAs. We speculate that many of the native PA discontinuities reported in other studies are due to spontaneous occlusion of tiny central PAs with time and hypoperfusion. During the study period, we observed only 1 infant with hypoplastic discontinuous PAs and MAPCAs and 1 patient with only MAPCAs and no native PAs. Both patients underwent successful surgical repair with unifocalization. They had a poor hemodynamic result. One of them eventually died.

\section{CONCLUSIONS}

For this difficult-to-treat group of patients, medicosurgical rehabilitation of the PAs with well-defined steps is successful, allowing complete surgical repair with good survival and functional status in the majority of the cases. Rehabilitation of PAs has to continue after the surgical correction with further interventional catheterization or surgical reintervention.

\section{References}

1. Tchervenkov CI, Salasidis G, Cecere R, Béland MJ, Jutras L, Paquet M, et al One-stage midline unifocalization and complete repair in infancy versus multiple-stage unifocalization followed by repair for complex heart disease with major aortopulmonary collaterals. J Thorac Cardiovasc Surg. 1997;114: 727-35.

2. Faller K, Haworth SG, Taylor JF, Macartney FJ. Duplicate sources of pulmonary blood supply in pulmonary atresia with ventricular septal defect. Br Heart J. 1981;46:263-8.

3. Duncan BW, Mee RB, Prieto LR, Rosenthal GL, Mesia CI, Qureshi A, et al. Staged repair of tetralogy of Fallot with pulmonary atresia and major aortopulmonary collateral arteries. J Thorac Cardiovasc Surg. 2003;126:694-702.

4. Reddy VM, Liddicoat JR, Hanley FL. Midline one-stage complete unifocalization and repair of pulmonary atresia with ventricular septal defect and major aortopulmonary collaterals. J Thorac Cardiovasc Surg. 1995;109:832-44.

5. d'Udekem Y, Alphonso N, Norgaard MA, Cochrane AD, Grigg LE, Wilkinson JL, et al. Pulmonary atresia with ventricular septal defects and major aortopulmonary collateral arteries: unifocalization brings no long-term benefits. J Thorac Cardiovasc Surg. 2005;130:1496-502.

6. Norgaard MA, Alphonso N, Cochrane AD, Menahem S, Brizard CP d'Udekem Y. Major aorto-pulmonary collateral arteries of patients with pulmonary atresia and ventricular septal defect are dilated bronchial arteries. Eur J Cardiothorac Surg. 2006;29:653-8.

7. Mumtaz MA, Rosenthal G, Qureshi A, Prieto L, Preminger T, Lorber R, et al. Melbourne shunt promotes growth of diminutive central pulmonary arteries in patients with pulmonary atresia, ventricular septal defect, and systemic-topulmonary collateral arteries. Ann Thorac Surg. 2008;85:2079-83. 
8. Rome JJ, Mayer JE, Castaneda AR, Lock JE. Tetralogy of Fallot with pulmonary atresia. Rehabilitation of diminutive pulmonary arteries. Circulation. 1993;88: 1691-8.

9. Metras D, Chetaille P, Kreitmann B, Fraisse A, Ghez O, Riberi A. Pulmonary atresia with ventricular septal defect, extremely hypoplastic pulmonary arteries, major aorto-pulmonary collaterals. Eur J Cardiothorac Surg. 2001;20:590-6.

10. Schouvey S, Dragulescu A, Ghez O, Amedro P, Kreitmann B, Chetaille P, et al. Rehabilitation of hypoplastic pulmonary arteries in pulmonary atresia with ventricular septal defect. Medium term results. Arch Mal Coeur Vaiss. 2007;100: 422-7.

11. Nakata S, Imai Y, Takanashi Y, Kurosawa H, Tezuka K, Nakazawa M, et al. A new method for the quantitative standardization of cross-sectional areas of the pulmonary arteries in congenital heart diseases with decreased pulmonary blood flow. J Thorac Cardiovasc Surg. 1984;88:610-9.

12. Rothman A, Perry SB, Keane JF, Lock JE. Early results and follow-up of balloon angioplasty for branch pulmonary artery stenosis. J Am Coll Cardiol. 1990;15: 1109-17.

13. Bergersen LJ, Perry SB, Lock JE. Effect of cutting balloon angioplasty on resistant pulmonary artery stenosis. Am J Cardiol. 2003;91:185-9.

14. Abella RF, De La TT, Mastropietro G, Morici N, Cipriani A, Marcelletti C. Primary repair of pulmonary atresia with ventricular septal defect and major aortopulmonary collaterals: a useful approach. J Thorac Cardiovasc Surg. 2004;127: 193-202.

15. Reddy VM, McElhinney DB, Amin Z, Moore P, Parry AJ, Teitel DF, et al. Early and intermediate outcomes after repair of pulmonary atresia with ventricular septal defect and major aortopulmonary collateral arteries: experience with 85 patients. Circulation. 2000;101:1826-32.

16. Chowdhury UK, Dipl NB, Bishnoi AK, Kalaivani M, Kapoor PM, Reddy SM, et al. Central pulmonary artery histopathology in patients with cyanotic congenital heart disease. Ann Thorac Surg. 2009;87:589-96.

17. Rosales AM, Lock JE, Perry SB, Geggel RL. Interventional management of perioperative peripheral pulmonary stenosis: balloon angioplasty or endovascular stenting. Cathet Cardiovasc Interv. 2002;56:272-7.

18. Marshall AC, Love BA, Lang P, Jonas RA, del Nido PJ, Mayer JE, et al. Staged repair of tetralogy of Fallot and diminutive pulmonary arteries with a fenestrated ventricular septal defect patch. J Thorac Cardiovasc Surg. 2003;126:1427-33.

19. Cho JM, Puga FJ, Danielson GK, Dearani JA, Mair DD, Hagler DJ, et al. Early and long-term results of the surgical treatment of tetralogy of Fallot with pulmonary atresia, with or without major aortopulmonary collateral arteries. J Thorac Cardiovasc Surg. 2002;124:70-81.

20. Sutton NJ, Peng L, Lock JE, Lang P, Marx GR, Curran TJ, et al. Effect of pulmonary artery angioplasty on exercise function after repair of tetralogy of Fallot. Am Heart J. 2008;155:182-6.

21. McMahon CJ, El-Said HG, Grifka RG, Fraley JK, Nihill MR, Mullins CE. Redilation of endovascular stents in congenital heart disease: factors implicated in the development of restenosis and neointimal proliferation. J Am Coll Cardiol. 2001; 38:521-6.

22. Liao PK, Edwards WD, Julsrud PR, Puga FJ, Danielson GK, Feldt RH. Pulmonary blood supply in patients with pulmonary atresia and ventricular septal defect. J Am Coll Cardiol. 1985;6:1143-50. 